\title{
Galectin-3 increases the motility of mouse melanoma cells by regulating matrix metalloproteinase-1 expression
}

\author{
Yuan-Guo Wang ${ }^{1}$, Seok-Jun Kim ${ }^{2,3,4}$, \\ Jung-Hwan Baek ${ }^{4,5}$, Hyun-Woo Lee ${ }^{4}$, \\ Seo-Young Jeong ${ }^{1}$ and Kyung-Hee Chun ${ }^{4,5,6}$ \\ ${ }^{1}$ Department of Practical Pharmacy \\ College of Pharmacy \\ Kyung Hee University \\ Seoul 130-701, Korea \\ ${ }^{2}$ Cancer Genomics Branch \\ Division of Convergence Technology \\ National Cancer Center Research Institute and Hospital \\ Goyang 410-769, Korea \\ ${ }^{3}$ Department of Biological Science \\ Sungkyunkwan University \\ Suwon 440-746, Korea \\ ${ }^{4}$ Department of Biochemistry and Molecular Biology \\ Yonsei University College of Medicine \\ Seoul 120-752, Korea \\ ${ }^{5}$ Brain Korea 21 Project for Medical Science of Yonsei University \\ ${ }^{6}$ Corresponding author: Tel, 82-2-2228-1699; \\ Fax, 82-2-312-5041; E-mail, khchun@yuhs.ac \\ http://dx.doi.org/10.3858/emm.2012.44.6.044
}

Accepted 12 March 2012

Available Online 21 March 2012

Abbreviations: ChIP, chromatin immunoprecipitation; IP, immunoprecipitation; MMP-1, matrix metalloproteinase-1

\begin{abstract}
Although mounting evidence indicates the involvement of galectin-3 in cancer progression and metastasis, the underlying molecular mechanisms remain largely unknown. In this study, we investigated the effect and possible mechanism of galectin-3 on the migration and invasion of $\mathrm{B} 16 \mathrm{~F} 10$, a metastatic melanoma cell line, in which galectin-3 and matrix metalloproteinase-1 (MMP-1) were both found to be highly expressed. Knockdown of galectin-3 with specific siRNA reduced migration and invasion, which was associated with reduced expression of MMP-1. To further investigate the underlying mechanism, we examined the effect of galectin-3 knockdown on the activity of AP-1, a transcriptional factor regulating MMP-1
\end{abstract}

expression. We found that galectin-3 directly interacted with AP-1 and facilitated the binding of this complex to the MMP-1 promoter that drives MMP-1 transcription. Moreover, silencing of galectin-3 inhibited binding of fra- 1 and c-Jun to promoter sites of MMP-1 gene. Consistent with these in vitro findings, our in vivo study demonstrated that galectin-3 shRNA treatment significantly reduced the total number of mouse lung metastatic nodules. Taken together, galectin- 3 facilitates cell migration and invasion in melanoma in vitro and can induce metastasis in vivo, in part through, regulating the transcription activity of AP-1 and thereby up-regulating MMP-1 expression.

Keywords: galectin 3; matrix metalloproteinase 1; melanoma; neoplasm metastasis; RNA, small interfering; transcription factor AP-1

\section{Introduction}

Galectin-3, a chimera-type of 31-kDa galactosebinding protein, is highly expressed in a variety of primary and metastatic tumors (Raz et al., 1990). Galectin-3 is associated with enhanced tumor progression, invasion and metastasis in various cancer cells, e.g., lung and gastric cancer cells (Hood and Cheresh, 2002; Liu and Rabinovich, 2005). Changes in circulating galectin-3 levels in cancer patients may reflect the metastatic spread of disseminating cancer cells (Yu, 2010). Thus, inhibition of galectin-3 may be a novel therapeutic approach for preventing metastasis and reducing cancer-associated mortality (Kim et al., 2010). However, the mechanisms underlying the role of galectin-3 in tumor progression and metastasis remain largely unclear.

Matrix metalloproteinases (MMPs) facilitate tumor progression and metastasis (Khokha, 1994; Ahonen et al., 1998; Valente et al., 1998). Among these, MMP-1, MMP-2, MMP-9 and MT1-MMP are involved in human melanoma invasion (Walker and Woolley, 1999; Hofmann et al., 2005). MMP-1, which is capable of degrading type I, II and III collagens, is the only MMP subtype able to degrade triple helical collagens (Pardo and Selman, 2005). Recent 
A

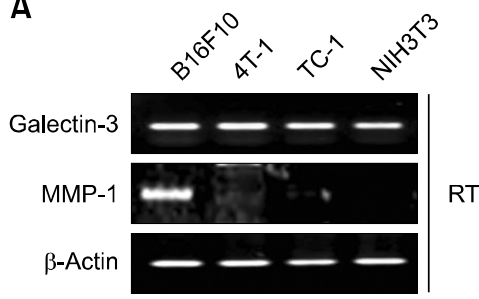

C

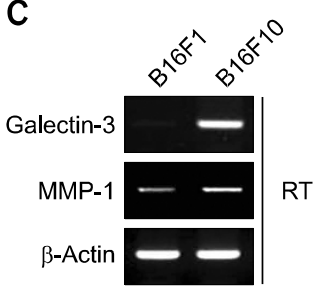

B

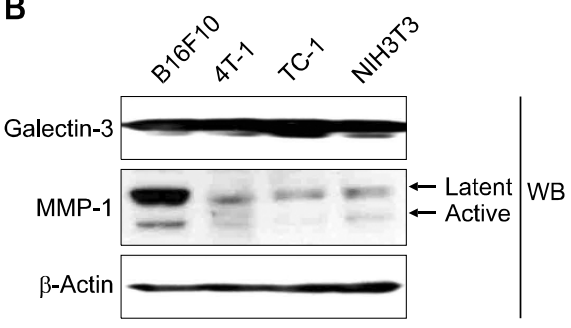

D

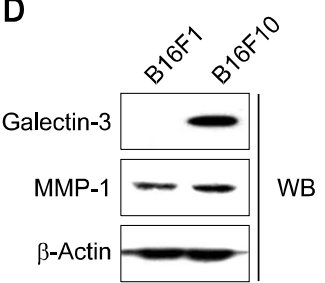

Figure 1. Expression levels of galectin-3 and MMP-1 in several mouse cell lines. (A) mRNA and (B) protein expression levels of galectin-3 and MMP-1 in B16F10, 4T-1,TC-1 and NIH3T3 cell lines, determined by RT-PCR and western blotting. $\beta$-actin was used as a loading control. (C) mRNA and (D) protein expression levels of galectin-3 and MMP-1 in B16F1 and B16F10 cell lines were determined by RT-PCR and western blotting. $\beta$-actin was used as normalization control. studies showed that MMP-1 knockdown cells reduce melanoma invasion into matrigel, whereas its activation promoted melanoma cell invasion into three dimensional type I collagen (Durko et al., 1997; Benbow et al., 1999). Clinical studies have also provided evidence that MMP-1 is positively correlated with malignancy of melanoma cells and poor prognosis of patients (Nikkola et al., 2002).

MMP-1 expression is controlled by mitogen activated protein kinase (MAPK) pathway(s) and the upstream transcription factor, activation of activator protein-1 (AP-1) (Tower et al., 2003; Jung et al., 2008). Although increasing attention has been paid to the roles of galectin-3 and MMP-1 in cancer metastasis, and a recent study showed a significant role of galectin-3 in MMP-1 expression in gastric cancer cells (Cheong et al., 2010; Cho et al., 2011; Kim et al., 2011), it is unclear whether similar regulation occurs in melanoma.

In this study, we demonstrated that galectin-3 regulated MMP-1 expression and melanoma cell metastasis using B16F10 cell line, as a highly expression of both galectin-3 and MMP-1 in a mouse melanoma cell line. The B16F10 cell line is well established in in vivo mouse lung metastasis systems (Nguyen et al., 2007). We demonstrated that knockdown of galectin-3 reduced MMP-1 expression and melanoma metastasis both in vitro and in vivo, in part by modulating AP-1 activity.

\section{Results}

\section{Expression levels of galectin-3 and MMP-1 in mouse cancer cell lines}

The mRNA and protein expression of galectin- 3 and MMP-1 were assessed in the following 4 cell types: highly metastatic melanoma cancer cell (B16F10), highly metastatic breast cancer cell (4T-1), HPV positive and invasive mouse cervical cancer cells (TC-1), and mouse fibroblast cells (NIH3T3). As shown in Figures $1 \mathrm{~A}$ and $1 \mathrm{~B}$, galectin-3 was highly expressed in all four cell lines, whereas the expression of MMP-1 was obviously higher in the B16F10 cell line than in others. Compared to the non-metastatic cell line B16F1, B16F10 cells express a higher level of both galectin-3 and MMP-1, in both mRNA and protein levels, respectively (Figures $1 \mathrm{C}$ and 1D).

\section{Galectin-3 knockdown inhibited B16F10 melanoma cell migration and invasion}

To examine the effects of galectin- 3 knockdown on B16F10 cell migration and invasion, cells were transfected with various concentrations of galectin-3 siRNA, and galectin-3 expression was assessed using real time PCR and western blotting respectively. As shown in Figure $2 \mathrm{~A}$, all three concentrations of galectin-3 siRNA significantly reduced protein expression, with no significant differences in potency. Thus, $20 \mathrm{nM}$ of galectin-3 was chosen for the following experiments. After transfection of B16F10 cells with $20 \mathrm{nM}$ galectin- 3 siRNA for $24 \mathrm{~h}$, the living cells were collected and subjected to wound healing, invasion and migration assays. As shown in Figure 2B, cells transfected with galectin-3 siRNA migrated significantly slower than those without transfection or transfected with a scRNA in wound healing assays. Representative images are shown in Figure 2C. In addition, our trans-well migration and invasion assays also demonstrated approximately $50 \%$ and $65 \%$ decrease in cell migration and invasion, respectively, in the galectin-3 siRNA transfected cells compared to the control cells (Figures 2D and 2E). 
A

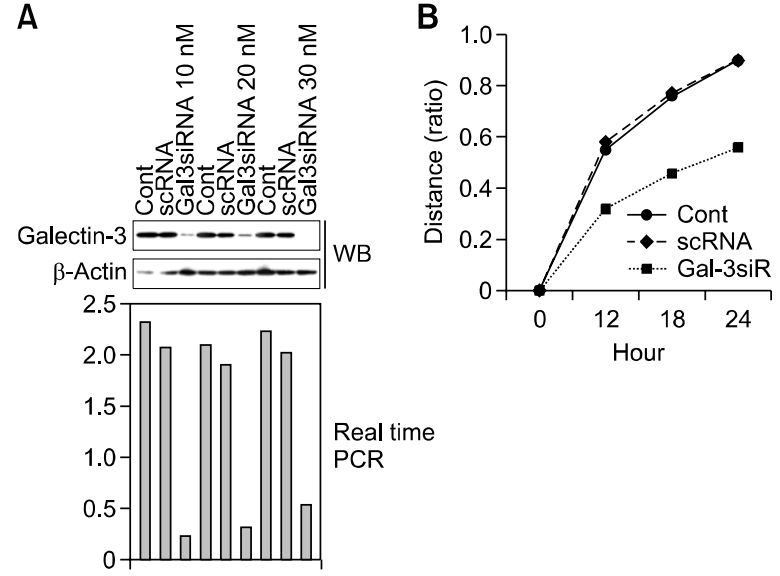

C
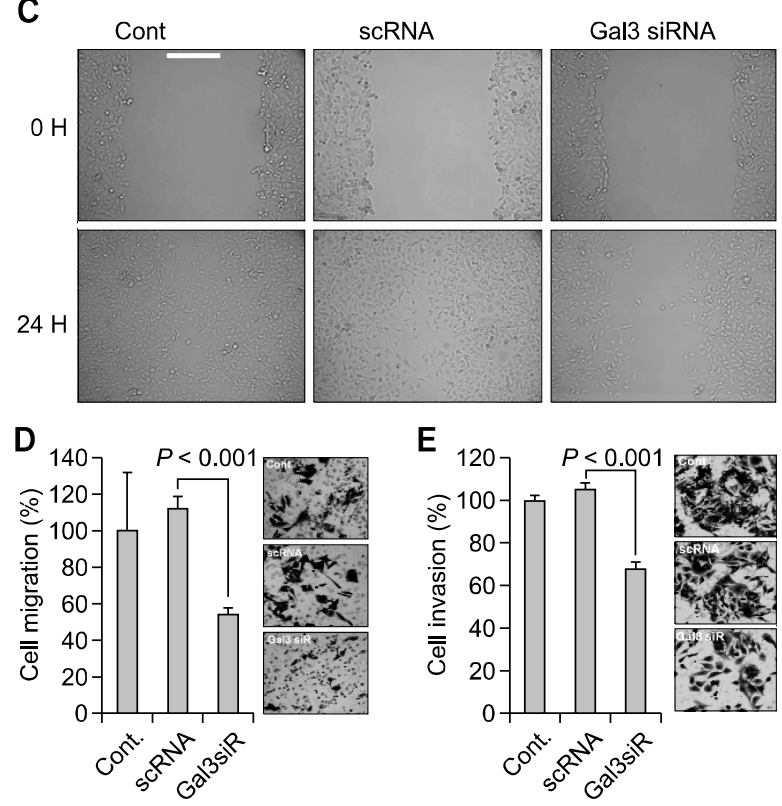

Figure 2. Effect of galectin-3 silencing on the migration and invasion of B16F10 melanoma cells. (A) mRNA and protein levels of galectin-3 after transfection of B16F10 melanoma cells with different concentrations (10, $20,30 \mathrm{nmol} / \mathrm{L}$ ) of scrambled siRNA (scRNA, negative control) and galectin-3 siRNA. Total RNA and protein were obtained after transfection for $48 \mathrm{~h}$, and cells were harvested and analyzed by real time-PCR and western blotting, respectively. $\beta$-actin was used as a loading control. (B-C) A wound healing assay of B16F10 cells was performed with scRNA or galectin-3 siRNA at 0, 12, 18 and $24 \mathrm{~h}$ after scratching. (B) Quantification of cell migration as described in the Methods section and $(C)$ the micrographs of B16F10 cells were obtained according to the width change of the area lacking cells (Bar $=100 \mu \mathrm{m})$. (D-E) Cell migration and invasion activities were measured respectively by a Transwell assay after transfection of melanoma cells with scRNA or galectin-3 siRNA for $24 \mathrm{~h}$. All experiments were performed individually in triplicate $(P<0.001$ vs. control group).

\section{Galectin-3 modulated MMP-1 expression through interacting with AP-1}

Given the expressions of both galectin-3 and MMP-1 in the mouse B16F10 cell line, we examined their possible interactions and the effect of MMP-1 on cell motility. MMP-1 knockdown had no effect on the expression of galectin-3, but galectin-3 knockdown resulted a marked reduction of MMP-1 expression, indicating that galectin-3 is an upstream regulator of MMP-1 (Figure 3A). A cell proliferation assay demonstrated that transfection of gal-3 siRNA or MMP-1 siRNA slightly decreased cell proliferation compared to the scrambled siRNA transfected cells (Figure 3B). In contrast, MMP-1 knockdown dramatically decreased cell motility (Figures 3C and 3D). To further explore how galectin-3 increases MMP-1 expression in melanoma cells, we focused on the transcription factor, activator protein-1 (AP-1), which is a dimer composed of Jun and Fos-like region antigen (Fra-1) (Angel and Karin, 1991). The direct interaction between galectin-3 and members of AP-1, C-Jun and Fra-1 was demonstrated by immunoprecipitation (Figure 3E). Next, we assessed transcription activity of AP-1 using a luciferase assay. Compared to the control cells, transfection of the cells with galectin-3 shRNA significantly reduced AP-1 transcription activity (Figure 3F). Finally, we examined DNA binding activity of AP-1 with or without galectin-3. Figure 3G shows the binding site of the AP-1 complex on the MMP-1 promoter region. Our ChIP assay demonstrated that $\mathrm{C}$-jun and fra-1 bound to the promoter regions of MMP-1 in the presence, but not in the absence, of galectin-3 (Figure $3 \mathrm{H}$ ).

\section{Galectin-3 knockdown decreased B16F10 metastasis in lung metastasis mouse models}

We constructed lentiviral vectors containing lac $Z$ (negative control) or galectin-3 shRNA, and infected $\mathrm{B} 16 \mathrm{~F} 10$ cells with the lentivirus. As shown in Figure $4 \mathrm{~A}$, transfection of the B16F10 cells with galectin-3 siRNA resulted in approximately $53 \%$ reduction of the mRNA level of the target protein. As expected, transfection of $\mathrm{B} 16 \mathrm{~F} 10$ cells with galectin- 3 markedly reduced the expression of MMP-1 (Figure 4B). To examine the effect of galectin-3 knockdown on melanoma metastasis in vivo, we applied the lentiviral infected cells to a mouse pulmonary metastasis model. Metastasis was assessed in C57BL/6 mice four weeks after tail vein injection of B16F10 parent cells, B16F10 cells infected with lentiviral lacZ, or gal-3shRNA. As shown in Figures 4C and 4D, the total numbers of lung metastatic nodule were reduced by approximately $50 \%$ in the animals injected with the B16F10 cells containing lentiviral gal-3 shRNA compared to the animals injected with B16F10 parent cells or lacZ expressing B16F10 cells. 
A
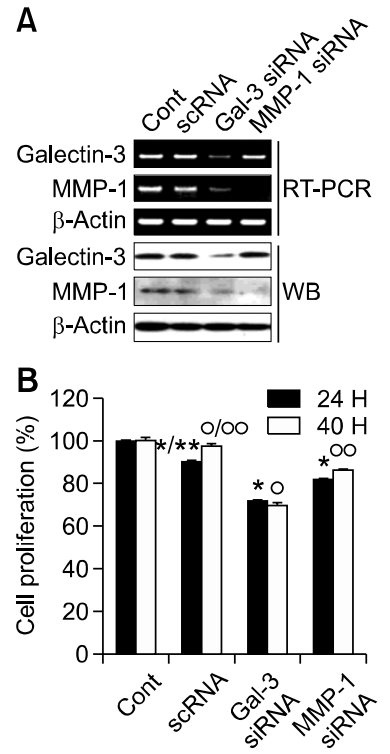

E

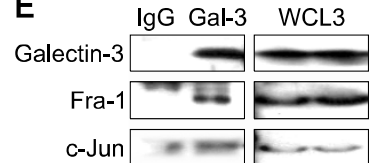

F

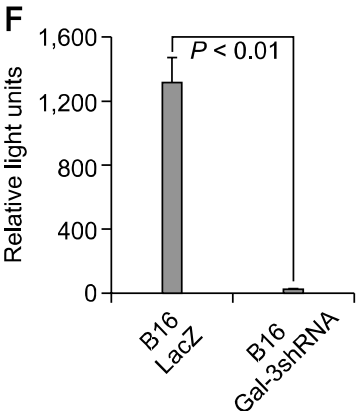

C

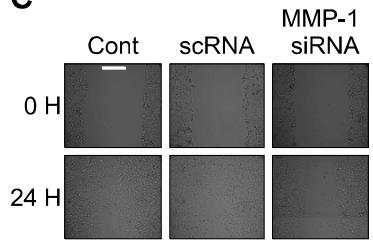

$D_{10} \rightarrow$ Cont

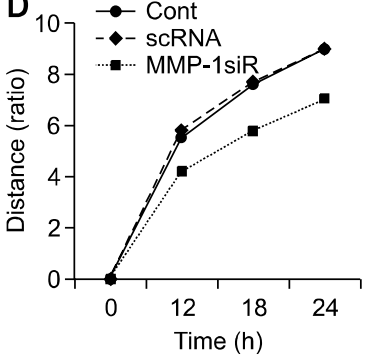

G

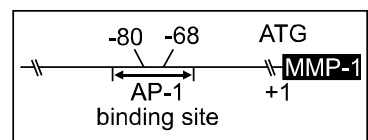

H

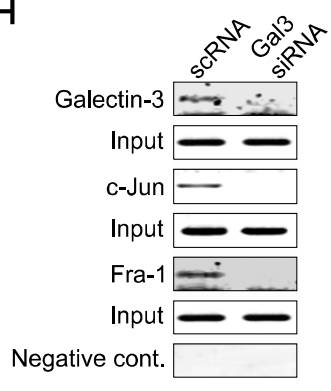

Figure 3. Effect of galectin-3 on MMP-1 expression through binding with AP-1 complex and effect of MMP-1 silencing on the motility of B16F10 melanoma cells. (A) mRNA and protein expression levels of galectin-3 or MMP-1 were detected by RT-PCR and western blotting respectively after transfection of B16F10 melanoma cells with galectin-3 or MMP-1 siRNA. scRNA was used as negative control and $\beta$-actin was used as a loading control. (B) Cell proliferation was detected after transfection of B16F10 cells with scrambled siRNA, galectin-3 siRNA or MMP-1 siRNA for 24 and $40 \mathrm{~h}$ by MTT assay as described in the Methods section ( ${ }^{*} P<0.001$, ${ }^{* *} P<$ $0.001, \circ P<0.001, \circ \circ P<0.001$ ). (C, D) Wound healing assays utilizing B16F10 melanoma cells were performed by scRNA or MMP-1 siRNA transfected cells at 0, 12, 18 and $24 \mathrm{~h}$ after creating a scratch. (C) The phase micrographs of B16F10 cells were obtained according to the data. (bar=100 $\mu \mathrm{m})$ and (D) quantification of cell migration using the monolayer wound healing assay was performed as described in the Methods section. All experiments were performed individually in triplicate. (E) Interaction between galectin-3 and AP-1 (Fra-1 and C-Jun) was measured by immunoprecipitation as previously described in "Methods", and then expression of galectin-3, Fra-1 and c-Jun was detected by western blot. Whole-cell lysates (WCLs) were used as a positive control. (F) Luciferase activity of AP-1 in lacZ and galectin-3 knock down cells. Luciferase assay was performed using an AP-1 expression luciferase vector transfection to lacZ and galectin-3 knock down cells ( $P<0.01$, vs. Cont). (G) The proximal AP-1 binding site which is located between -80 and -68 from the initiation codon of the MMP-1 gene. $(\mathrm{H}) \mathrm{AChlP}$ assay was performed using antibodies against galectin-3, C-Jun and Fra-1 in melanoma cells transfected with scRNA and galectin-3 siRNA. In the input lane, total genomic DNA was used as a control for the PCR reaction.
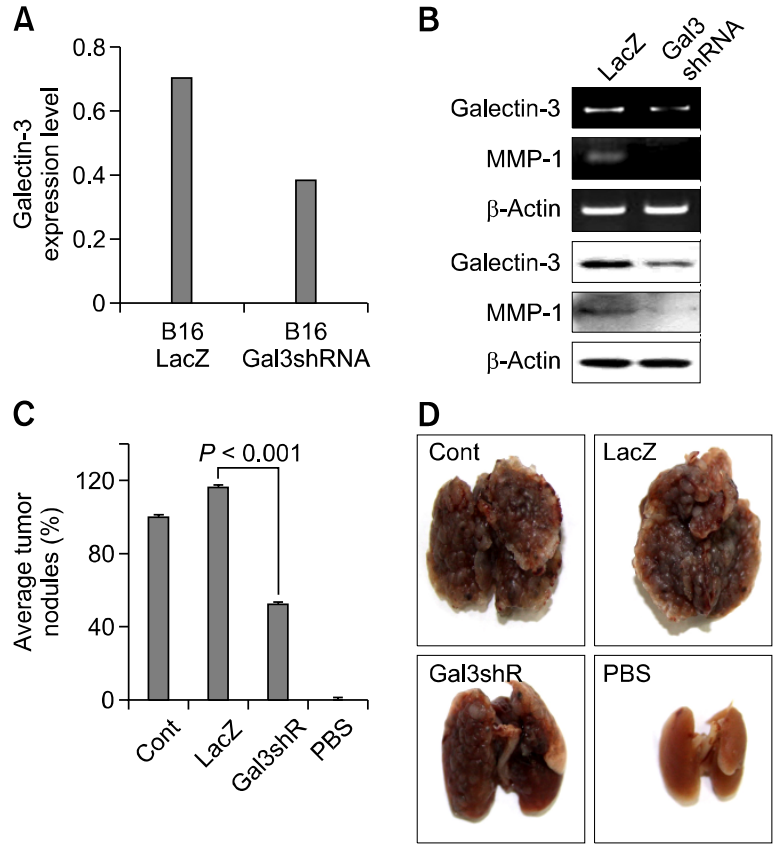

Figure 4. Suppressive effects of galectin-3 silencing on metastasis of B16F10 melanoma cells in lung metastasis mouse models. (A) Expression levels of galectin-3 were detected by real time-PCR analysis in B16F10 cells infected with lenti-virus expressing LacZ or galectin-3 shRNA (gal-3shRNA). The preparation and infection of these lenti-viruses were described in the Methods section. (B) Expression levels of galectin-3 and MMP-1 were detected by RT-PCR and western blot analysis in B16F10 melanoma cells transfected stably with lacZ and gal-3shRNA respectively. $\beta$-actin was used as a loading control. (C, D) Four weeks after tail vein injection of PBS (negative control), parent B16F10 melanoma cells, lacZ expressing B16F10 cells or gal-3shRNA expressing cells into $\mathrm{C} 57 \mathrm{BL} / 6$ mice, the formation of lung colonies was counted and illustrated as a histogram ( $P<0.01$ vs. lacZ group) (C) and photographs (D). The details are described in the Methods section.

\section{Discussion}

This study was performed to better understand the mechanisms underlying the role of galectin-3 in melanoma invasion and metastasis. We have previously shown that after silencing of galectin- 3 , some genes such as MMP-1 and MMP-3 are down-regulated, while other genes, such as serine protease inhibitor and tissue inhibitors of MMP-2 were up-regulated (data not shown) (Cheong et al., 2010). Among these genes, MMP-1 was downregulated by galectin-3 to a greater extent than others, which was further confirmed by the following findingsWe confirmed the extensive expression of galectin-3 in different mouse cell lines including B16F1, B16F10 (melanoma), 4T-1 (breast carcinoma), TC-1 (lung cancer) and NIH3T3 (embryonic fibroblast), which may imply its conservation and significance in many types of cancer. However, much higher expression of MMP-1 was detected in the melanoma cell line B16F10 than in the 4T-1, 
TC-1 and NIH3T3 cell lines. We also assessed galectin-3 and MMP-1 expression in the B16F1 and B16F10 melanoma cell lines and found that the non-metastatic cell line (B16F1) did not express galectin-3 while the metastatic cell line (B16F10) did. A previous report indicated that galectin-3 regulates the metastasis of various types of cancer via different mechanisms (Liu and Rabinovich, 2005). However, the mechanisms underlying the involvement of galectin-3 in mouse cancer cell motility remain largely unknown. Our findings seem to indicate that one possible mechanism of melanoma cell motility in mice involves a putative relationship between galectin-3 and MMP-1 expression.

We observed that galectin-3 knockdown dramatically suppressed the motility, migration, and invasion of melanoma cells. In parallel, MMP-1 knockdown also significantly reduced the cell motility. Knockdown of galectin-3 reduced the expression of MMP-1 but not vice versa, and it is suggested that galectin-3 is an upstream regulator of MMP-1. This finding sheds new light on the mysterious relationships between galectin- 3 and MMP-1. To understand how galectin-3 regulates MMP-1 expression, we determined the interaction between galectin-3 and the AP-1 complex (Song et al., 2005). The AP-1 family of transcription factors is composed of the homodimers or heterodimers of the Jun (JunB, JunD, c-Jun) and Fos (c-Fos, Fos B, fra-1, fra-2) onco-proteins (Lee et al., 1987). Among them, c-Jun plays important roles in regulating cancer invasion and metastasis. For instance, the c-Jun dominant-negative mutant, TAM67, inhibits the effects of AP-1 on the growth of breast cancer (Moore-Carrasco et al., 2006), as well as on the invasion and metastasis of murine osteosarcomas (Leaner et al., 2009). Fra-1, another component of the AP-1 family, plays a key role in MMP-1 transcription in A2058 melanoma cells (Tower et al., 2003). Here, we provide evidence for the association of galectin-3 with c-Jun and fra-1 and regulation of AP-1 transcription activity. Interestingly, C-Jun and fra- 1 bound to the promoter regions of MMP-1 in the presence of galectin-3, but not in its absence. This is the first case reported in which galectin-3 contributes to MMP-1 promoter activity by forming a complex with c-Jun and fra-1 in melanoma cells. The role of galectin- 3 in melanoma metastasis was also supported by the in vivo experimental results. In a mouse pulmonary metastasis model, animals treated with galectin-3 shRNA-transfected B16F10 melanoma cells showed considerable inhibition of lung metastasis compared with the untreated control group.

In conclusion, the promotion of galectin-3 expression resulted in melanoma invasion/metastasis due at least in part to increased MMP-1 expression via the transcriptional activation of AP- 1 . These findings combined with the recent report by Kim et al, for the involvement of galectin-3 in gastric cancer cell motility (Kim et al., 2011), suggest that galectin-3 plays a role in various cancers' metastasis through a common molecular mechanism.

\section{Methods}

\section{Cell culture and siRNAs transfection}

B16F1, B16F10, 4T-1, TC-1 and NIH3T3 cell lines obtained from the Korea Cell Line Bank were cultured in DMEM or RPMI 1640 medium with 5\% fetal bovine serum (FBS) and 1\% Antibiotics (Invitrogen). Both galectin-3 and MMP-1 siRNAs were purchased from Invitrogen and transfection was performed with Lipofectamine RNAiMAX reagent (Invitrogen) following the manufacturer's instructions. The sequences of mouse galectin- 3 and MMP- 1 siRNAs were 5'-AUGAUUGUGAUCAGCAUGCTT-3' and 5'-GCCA GAACTTCCCAACCAT-3', respectively.

\section{RNA isolation and RT-PCR}

Total RNA was extracted from mouse melanoma cells with TRIzol reagent (Invitrogen) according to the manufacturer's protocol. RT was carried out using a Reverse Transcription system (Promega), and PCR was performed with Ex-taq DNA polymerase (TaKaRa). The sequences of primers were as follows: mMMP-1; 5'-CCTGGAATTGGCAACAAAGT-3' (sense) and 5'-TAGCACGCAAGAATCAGGTG-3' (anti-sense); mGalectin-3: 5'-CAGTGCTCCTGGAGG CTATC-3' (sense) and 5'-AAGGGGAAGGCTGACTGTCT-3' (anti-sense); $\mathrm{m} \beta$ actin: 5'-AGCCTCGCCTTTGCCGA-3' (sense) and 5'-CTG GTGCCTGGGGCG-3' (anti-sense).

\section{Western blot analysis}

Western blot analysis was carried out as described previously (Kim et al., 2010). Briefly, cells were lysed in RIPA buffer (Biosesang) containing a protease inhibitor cocktail (Sigma), followed by sonication on ice. The cell lysate was centrifuged and the supernatant was collected. Twenty $\mu \mathrm{g}$ of proteins were subjected to SDS-PAGE and transferred to PVDF transfer membranes (Amersham). After being blocked with $5 \%$ skim milk for $1 \mathrm{~h}$, the membrane was incubated with primary antibody dissolved in $5 \%$ BSA overnight at $4^{\circ} \mathrm{C}$. The membrane was then incubated with secondary antibody for $1 \mathrm{~h}$, followed by detection with an ECL kit (Amersham). The following antibodies were used: anti- $\beta$-actin (Santa Cruz); anti-galectin-3 (Santa Cruz); anti-MMP-1 (Calbiochem, Santa Cruz).

\section{Immunoprecipitation assays}

Cell lysate containing $750 \mu \mathrm{g}$ of protein was pre-cleared by incubation with $40 \mu \mathrm{l}$ protein-A/G linked agarose beads (Santa Cruz) for $1 \mathrm{~h}$ at $4^{\circ} \mathrm{C}$. After the beads were spun 
down, the supernatant was incubated with $1 \mu \mathrm{g}$ specific antibody (anti-galectin-3, anti-c-Jun and anti-Fra-1, respectively) overnight at $4^{\circ} \mathrm{C}$, followed by incubation with $40 \mu \mathrm{l}$ protein-A/G agarose beads for $1 \mathrm{~h}$. Mouse/rabbit IgG (Santa Cruz) was used as the negative control. After the incubation, beads were washed 3 times in RIPA buffer before being dissolved in SDS-PAGE loading buffer. Western blot analysis was performed as described above.

\section{Transfilter migration and invasion assays}

Transfilter migration and invasion assays were carried out with $8.0-\mu \mathrm{m}$ pore inserts in 24-well Transwells (Corning Costar). Briefly, B16F10 cells were transfected with galectin-3 or MMP-1 siRNAs. One day after the transfection, cells were isolated and added to upper Transwell chambers with $0.5 \mathrm{mg} / \mathrm{ml}$ collagen type I (BD Bioscience) coated filters for the migration assay, and with 1/15 dilution of matrigel (BD Bioscience) coated filters for the invasion assays. DMEM containing $10 \%$ FBS and $1 \%$ antibiotics was added to the lower chamber and incubation continued for $20 \mathrm{~h}$. Cells that had migrated or invaded into the lower chamber were quantified after H\&E staining. For quantification, cells on were counted at 5 randomly selected areas in each well using a wide-field microscopy. Data were expressed as mean $\pm \mathrm{SD}$ from three independent experiments.

\section{Wound healing assays}

B16F10 cells were seeded into 6 -well culture dishes at $1 \times$ $10^{5}$ cells/well, and maintained in DMEM containing $5 \%$ FBS for $24 \mathrm{~h}$. The nearly confluent cells were scratched with a pipette tip and cellular debris was removed. The scratched monolayer was then incubated for another $24 \mathrm{~h}$ in DMEM. Cells migrating into the gap were visualized and counted under a microscope.

\section{Lentivial galectin-3 shRNA construction and stable cell line establishment}

Lenti-viral vectors containing galectin-3 shRNA (Gal-3shRNA) was constructed by inserting the double strand gal-3 shRNA into the lentiviral vector pLL3.7 as described previously (Kim et al., 2010). The sequence of the shRNA was as following: gal-3shRNA sense: 5'-TGAACAACAGGAGA GTCATTGTTTCAAGAGAACAATGACTCTCCTGTTGTTC TTTTTTC-3'; gal-3shRNA antisense: 5'-TCGAGAAAAAA GAACAACAGGAGAGTCATTGTTCTCTTGAAACAATGAC TCTCCTGTTGTTCA-3'. The lentiviral vector containing lacZ was used as a control. Lentiviral infection of B16F10 melanoma cells was performed as described previously (Kim et al., 2010). Approximately $80 \%$ cells were infected and confirmed with FACS analysis.

\section{Mouse pulmonary metastasis model}

Female C57BL/6 mice, purchased from Orient Bio in Korea, were maintained in the animal facility at the National Cancer Center (NCC). B16F10 cells $\left(1 \times 10^{6} \mathrm{cells} / \mathrm{ml}\right.$ in $100 \mu \mathrm{l}$ PBS) infected with lentiviral lacZ (control) or gal-3shRNA was injected into 6-week-old female C57BL/6 mice (five mice per group) through the lateral tail vein. Four weeks after the injection, mice were sacrificed with an overdose of anesthesia. The number of metastatic nodules on the surface of the lung was counted under the surgical microscope.

\section{Chromatin immunoprecipitation assay}

A chromatin immunoprecipitation (ChIP) assay was carried out using a ChIP assay kit (Upstate). Samples were applied to dishes after galectin-3 siRNA treatment and assays were conducted following the manufacturer's instructions. Anti-galectin-3, c-Jun and Fra-1 and normal mouse/rabbit IgG were used to immunoprecipitate DNA containing complexes. Prior to PCR, primers were prepared for AP-1 with MMP-1 promoter binding sites: MMP-1 promoter (-2950) 5'-AAGAAGAAGGTGGCCAGGAT-3' and (-2901) 5'-TGCCTTCATTT TCCATTTCC-3'. PCR was performed with Ex Taq (Takara)

\section{Cell proliferation assay}

B16F10 cells, growing in 96-well culture plates at a density of $3 \times 10^{3}$ cells/well, were transfected with scrambled siRNA, galectin-3 siRNA, and MMP-1 siRNA respectively. 24 or 40 -h post transfection, cell viability was analyzed by MTT assay, as described previously (Cheong et al., 2010).

\section{Luciferase assays}

B16F10 melanoma cell lines were co-transfected with an AP- 1 reporter plasmid and galectin-3 siRNA $48 \mathrm{~h}$ before the luciferase assay was performed, as described previously (Kim et al., 2010).

\section{Statistics analysis}

All data were obtained from at least three independent experiments and are presented as mean $\pm S D$, unless otherwise indicated. Statistical analysis was performed using one-way ANOVA. Data were considered significant if $P<$ 0.05 .

\section{Acknowledgements}

This study was supported by a grant of the Korea Health technology R\&D Project, Ministry of Health \& Welfare (A110961), Republic of Korea.

\section{References}

Ahonen M, Baker AH, Kahari VM. Adenovirus-mediated gene delivery of tissue inhibitor of metalloproteinases-3 inhibits invasion and induces apoptosis in melanoma cells. Cancer Res 1998;58:2310-5

Angel P, Karin M. The role of Jun, Fos and the AP-1 complex in cell-proliferation and transformation. Biochim Biophys Acta 1991;1072:129-57 
Benbow U, Schoenermark MP, Mitchell TI, Rutter JL, Shimokawa K, Nagase H, Brinckerhoff CE. A novel host/tumor cell interaction activates matrix metalloproteinase 1 and mediates invasion through type I collagen. J Biol Chem 1999;274:25371-8

Cheong TC, Shin JY, Chun KH. Silencing of galectin-3 changes the gene expression and augments the sensitivity of gastric cancer cells to chemotherapeutic agents. Cancer Sci 2010;101:94-102

Cho IR, Koh SS, Min HJ, Kim SJ, Lee Y, Park EH, Ratakorn $\mathrm{S}$, Jhun BH, Oh S, Johnston RN, Chung YH. Pancreatic adenocarcinoma up-regulated factor (PAUF) enhances the expression of beta-catenin, leading to a rapid proliferation of pancreatic cells. Exp Mol Med 2011;43:82-90

Durko M, Navab R, Shibata HR, Brodt P. Suppression of basement membrane type IV collagen degradation and cell invasion in human melanoma cells expressing an antisense RNA for MMP-1. Biochim Biophys Acta 1997;1356:271-80

Hofmann UB, Houben R, Brocker EB, Becker JC. Role of matrix metalloproteinases in melanoma cell invasion. Biochimie 2005;87:307-14

Hood JD, Cheresh DA. Role of integrins in cell invasion and migration. Nat Rev Cancer 2002;2:91-100

Jung E, Lee J, Huh S, Hwang H, Kim Y, Kim YW, Byun SY, Park D. Matrine inhibits PMA-induced MMP-1 expression in human dermal fibroblasts. Biofactors 2008;33:121-8

Khokha R. Suppression of the tumorigenic and metastatic abilities of murine B16-F10 melanoma cells in vivo by the overexpression of the tissue inhibitor of the metalloproteinases-1. J Natl Cancer Inst 1994;86:299-304

Kim SJ, Choi IJ, Cheong TC, Lee SJ, Lotan R, Park SH, Chun $\mathrm{KH}$. Galectin-3 increases gastric cancer cell motility by up-regulating fascin-1 expression. Gastroenterology 2010; 138:1035-45 e1-2

Kim SJ, Shin JY, Lee KD, Bae YK, Choi IJ, Park SH, Chun $\mathrm{KH}$. Galectin-3 facilitates cell motility in gastric cancer by up-regulating protease-activated receptor-1 (PAR-1) and matrix metalloproteinase-1 (MMP-1). PLoS One 2011;6: e25103

Leaner VD, Chick JF, Donninger H, Linniola I, Mendoza A, Khanna C, Birrer MJ. Inhibition of AP-1 transcriptional activity blocks the migration, invasion, and experimental metastasis of murine osteosarcoma. Am J Pathol 2009;174:265-75

Lee W, Mitchell P, Tjian R. Purified transcription factor AP-1 interacts with TPA-inducible enhancer elements. Cell 1987;49:741-52

Liu FT, Rabinovich GA. Galectins as modulators of tumour progression. Nat Rev Cancer 2005;5:29-41

Moore-Carrasco R, Garcia-Martinez C, Busquets S, Ametller E, Barreiro E, Lopez-Soriano FJ, Argiles JM. The AP-1/CJUN signaling cascade is involved in muscle differentiation: implications in muscle wasting during cancer cachexia. FEBS Lett 2006;580:691-6

Nguyen NQ, Cornet A, Blacher S, Tabruyn SP, Foidart JM, Noel A, Martial JA, Struman I. Inhibition of tumor growth and metastasis establishment by adenovirus-mediated gene transfer delivery of the antiangiogenic factor $16 \mathrm{~K} \mathrm{hPRL}$. Mol Ther 2007;15:2094-100

Nikkola J, Vihinen P, Vlaykova T, Hahka-Kemppinen M, Kahari VM, Pyrhonen S. High expression levels of collagenase-1 and stromelysin-1 correlate with shorter disease-free survival in human metastatic melanoma. Int $\mathrm{J}$ Cancer 2002;97:432-8

Pardo A, Selman M. MMP-1: the elder of the family. Int J Biochem Cell Biol 2005;37:283-8

Raz A, Zhu DG, Hogan V, Shah N, Raz T, Karkash R, Pazerini $\mathrm{G}$, Carmi P. Evidence for the role of $34-\mathrm{kDa}$ galactoside-binding lectin in transformation and metastasis. Int J Cancer 1990;46:871-7

Song S, Byrd JC, Mazurek N, Liu K, Koo JS, Bresalier RS. Galectin-3 modulates MUC2 mucin expression in human colon cancer cells at the level of transcription via AP-1 activation. Gastroenterology 2005;129:1581-91

Tower GB, Coon Cl, Belguise K, Chalbos D, Brinckerhoff CE. Fra-1 targets the AP-1 site/2G single nucleotide polymorphism (ETS site) in the MMP-1 promoter. Eur J Biochem 2003;270:4216-25

Valente P, Fassina G, Melchiori A, Masiello L, Cilli M, Vacca A, Onisto M, Santi L, Stetler-Stevenson WG, Albini A. TIMP-2 over-expression reduces invasion and angiogenesis and protects B16F10 melanoma cells from apoptosis. Int $\mathrm{J}$ Cancer 1998;75:246-53

Walker RA, Woolley DE. Immunolocalisation studies of matrix metalloproteinases-1, -2 and -3 in human melanoma. Virchows Arch 1999;435:574-9

Yu LG. Circulating galectin-3 in the bloodstream: An emerging promoter of cancer metastasis. World J Gastrointest Oncol 2010;2:177-80 